\title{
An Overview on Averaging for Pulse-modulated Switched Systems
}

\author{
Carmen Pedicini ${ }^{\natural}$, Francesco Vasca ${ }^{\natural}$, Luigi Iannelli ${ }^{\natural}$, Ulf Jonsson $\diamond$
}

\begin{abstract}
Averaging of fast switching systems is an effective technique used in many engineering applications. Practical stability and control design for a nonsmooth switched system can be inferred by analyzing the smooth averaged system. In this paper we overview the few formal approaches proposed in the literature to deal with the averaging of nonsmooth systems. The dithering, the phasor dynamics and the hybrid framework techniques are recast and compared by considering pulsemodulated switched linear systems as the common modeling platform.
\end{abstract}

\section{INTRODUCTION}

A switched system is a hybrid system consisting of a family of dynamical subsystems and a policy that at each time instant selects the active subsystem among a set of possible modes [1]. The selection policy is usually described by means of a switching function, say $\sigma(\cdot)$, which is a function of time and might also explicitly depend on the state and on the exogenous input. The potentially complex interactions between the modes dynamics and the high frequency switching signal complicates the analysis of switched models, also for deterministic systems which is the case considered in this paper. A possible approach to circumvent some of these difficulties is to average the hybrid dynamics over a suitable modulation period and to base the analysis and control design on the simpler averaged system.

The averaging of switched models has a wide practical interest for many engineering applications. Most power electronics systems have switching devices that, more or less instantaneously, change the dynamics of the system [2]. Ideal models of diodes and controlled switches give rise to a class of switched systems which consists of differential equations with discontinuous nonlinearities and external excitation signals. Averaging is a widely used technique in the power electronics community since 1970's [3], [4]. The averaging approach has been also applied to other switched systems of practical interest such as multi-agent systems [5], synchronization of oscillators [6], [7], pneumatic systems [8], switched controllers [9], [10], congestion control mechanism [11], robotic manipulators [12], and nonlinear circuits [13].

Averaging theory is a powerful tool to analyze nonlinear systems and has a rich history for smooth systems, for which it has been proved the closeness of the trajectories of the averaged system to the unaveraged system and the

(দ) Department of Engineering, University of Sannio, Piazza Roma 21, 82100 Benevento, Italy, email: \{carmen.pedicini, francesco.vasca, luigi.iannelli\}@unisannio.it

$(\diamond)$ We very much regret that our honoured colleague Ulf Jonsson died during the period of writing this paper. He was with Department of Mathematics, Royal Institute of Technology (KTH), Stockholm, Sweden. relationship between the stability properties of the two systems [14]. Averaging theory for switched systems has sofar attracted limited interest in the control literature and a common definition of the averaged model has not yet appeared. Similarly to the definition of averaging for smooth systems [14], the first definitions of averaged for nonperiodic nonsmooth systems were based over an infinite time horizon [15], [16]. Pulse-modulated switched systems, though till not being periodic, are usually characterized by switching functions with fast dynamics determined by a "small" modulation period. Making an analogy with the averaging for smooth systems, one should expect that the typical small parameter characterizing the approximation between the original system and the averaged one is somehow related to the switching function modulation period. Indeed the classical averaged definition for pulse-modulated systems is obtained by averaging the (periodic) switching function over a suitable modulation period [3]. For non-periodic switching functions the averaging can be carried out by considering a moving average over a modulation period time interval [17] [19]. Such definition can be generalized using frequency domain arguments, leading to the so-called dynamic phasor model [17], [20]. In order to take into account the exogenous input time-dependence in the averaging process, the notions of time invariant strong averaged system and of time-varying partial strong averaged system have been introduced in [21].

To the best of our knowledge the first averaging theorem for discontinuous time systems was presented in [15]; similar results were obtained later for impulsive differential equations [16]. The error bound due to the averaging of switched linear systems, i.e. switched systems with each mode representable as a linear time-invariant system, with two modes is analyzed in [22]. In the same paper a stability result of the error dynamics for multiple modes homogenous systems is also derived. By following a similar approach in [19] the results on the homogenous systems are extended by relating the stability of the averaged system to a maximum modulation period of the switched system.

An interesting alternative strategy to tackle the averaging problem consists in interpreting the switching function through the dithering technique. The basic principle of dithering is that if we add a suitable high frequency signal to the input of a nonlinearity, the averaged input-output relation is smoothed [23]. Of course this effect depends on the features of the dither signal. In [24] it is proved an averaging theorem for pulse-width modulated systems with triangular dither and in [25] similar results are obtained for the pulse-width modulated system with measurable or slowly varying inputs and in this case also stability conditions are given. In [26] 
and [27] the averaging theorem is extended to a class of switched systems with a quite arbitrary periodic dither.

Recently, a more general class of switched systems within the hybrid systems framework has been studied with the purpose to apply averaging to this type of systems [28], in this case an explicit two-time scale separation approach is used. The separation between fast and slow time scales is also used for the stability analysis in [29]. A different discussion should be dedicated to the averaging analysis for stochastic systems [30]-[34] and differential inclusions [35], which are not considered herein.

In this paper we overview the major results on averaging for switched systems for which fast and finite switchings among different modes is a natural behavior. In that sense, inspired by several practical applications, we exclude the presence of Zeno solutions, sliding modes and state jumps. Moreover for the sake of simplicity we consider switched linear systems. This choice is also motivated by the goal of highlighting some problems which still need a solution on averaging for such simple class of switched systems. However, most of the results presented can be applied also to switched Lipschitz systems. We recast phasor dynamics, dithering and hybrid systems approaches into this common framework, highlighting the existing results and some open problems which could suggest possible directions for future research.

\section{HigH FREQUENCY MODULATED SWITCHED SYSTEMS}

A switched linear system [1] is described by the equation

$$
\dot{x}(t)=A_{\sigma(t)} x(t)+B_{\sigma(t)} w(t)
$$

with the state $x \in \mathbb{R}^{n}$, the exogenous input $w \in \mathbb{R}^{p}$ (called 'disturbance' by some authors) and the switching function $\sigma(t):[0,+\infty) \rightarrow \Sigma$ being a piecewise constant, right-continuous function which selects at each time instant $t$ the index of the active mode from the finite index set $\Sigma=\{1, \ldots, m\}$. In (1) we assume that the state vector is the same for all modes. That might become a critical assumption if a minimal representation for each mode is required.

The model (1) has an interesting alternative representation. Consider an invertible map that associates each element $\sigma \in \Sigma$ to a vector $q=\left(q_{1}, \ldots, q_{m}\right)^{T}$ with $q_{i}=1$ for $i=\sigma$ and $q_{i}=0$ for $i \neq \sigma$. Then, the switching function $\sigma(t)$ corresponds to the piecewise constant function $q(t):[0, \infty) \rightarrow\{0,1\}^{m}$ with

$$
\sum_{i=1}^{m} q_{i}(t)=1, \quad \forall t \in[0, \infty) .
$$

A possible representation of $\sigma(t)$ and of the components $q_{i}(t)$ is reported in Fig. 1. Since $\sigma(t)$ and $q(t)$ are equivalent, without ambiguity also $q(t)$ will be called the switching function. The model (1) can then be represented in the equivalent form

$$
\dot{x}(t)=\sum_{i=1}^{m} q_{i}(t)\left(A_{i} x(t)+B_{i} w(t)\right) .
$$

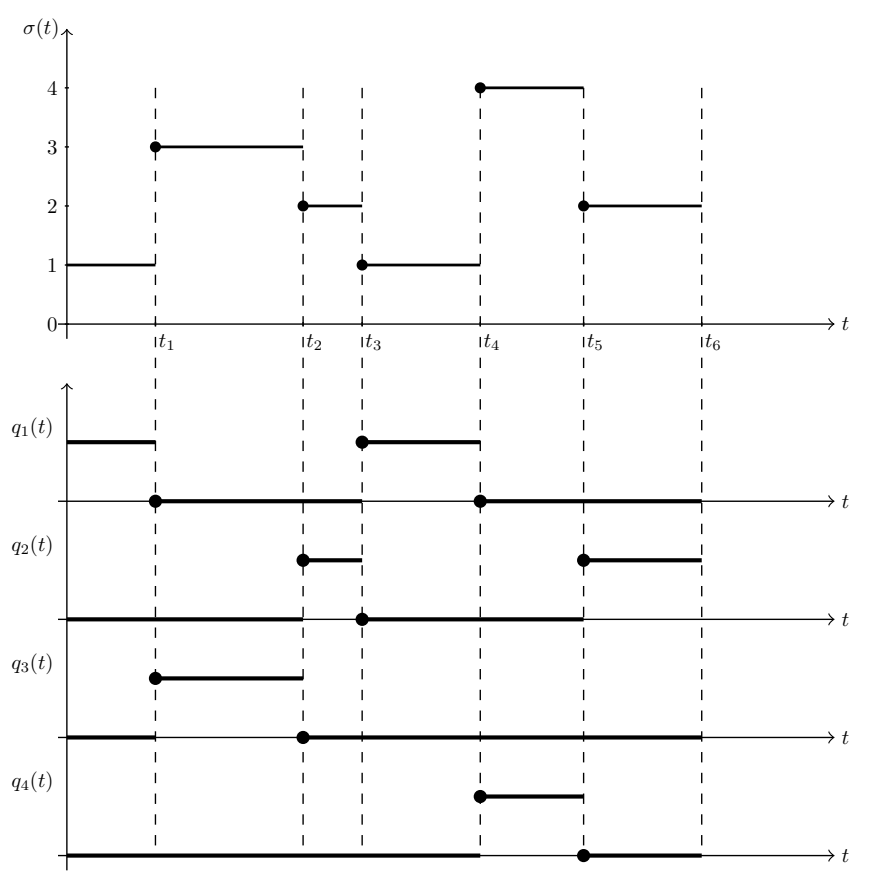

Fig. 1. Switching signals in the case of four modes.

First of all we need to define the solution concept. Given the initial condition $x(0)=x_{0}$, by a solution of the switched system (3) we mean a pair $(x(t), q(t))$ with $x(t)$ absolutely continuous, that satisfies (3) for almost all $t \in[0,+\infty)$.

At the isolated switching time instants, i.e. the time instants at witch the switching functions have discontinuities, the state time derivative is not well defined in the classical sense. A typical behavior of switched systems corresponds to a repetitive occurrence of the different modes over time. In order to relate such property with the averaging technique we need the definition of cycle and cycle-time. A first approach considers as cycle any sequence of modes in which each mode appears at most once. Given $\sigma(t)$, any sequence of modes can be represented as a sequence of cycles. For instance, for $m=4$ and $\sigma(t)$ determining the following timesequence of modes 1234123212314 , then the corresponding (unique) sequence of cycles will be $\{1234,123,21,2314\}$. Consider a sequence of modes; without loss of generality we denote $t_{k}^{i n}$ the time instant at which the $k$ th cycle starts and $t_{k+1}^{i n}$ the time instant when the $k$ th cycle ends and the $(k+1)$ th cycle starts. The time interval $t_{k+1}^{i n}-t_{k}^{i n}=T_{k}$ is called cycle-time of the $k$ th cycle. The duration of a mode $i$ in the $k$ th cycle is denoted by $\tau_{i, k}$. The fraction of cycle-time that the system spends in that mode is called duty ratio $d_{i, k}$ :

$$
d_{i, k} \triangleq \frac{\tau_{i, k}}{T_{k}}
$$

Clearly we have $d_{i, k} \in[0,1]$ for any $i$ and $k$ and

$$
\sum_{i=1}^{m} d_{i, k}=1 \text {. }
$$

If $d_{i, k}=0$ for some $i=M$ then the mode $\sigma=M$ is not visited in the $k$ th cycle-time. Some authors define 
the cycle and correspondingly the cycle-time depending on the specific mode. In such framework the cycle-time is given by the time interval $T_{i, k}$ between the $k$ th and the $(k+1)$ th occurrences of the $i$ th mode. An alternative and most common approach defines the cycle-time as a constant $T$ independent of the sequence of modes and related to some exogenous periodic signal. For instance this the case of pulsewidth modulated and pulse-phase modulated systems [9]. Note that the assumption of a constant cycle-time, which is quite common in practical switched systems, does not imply that the system is periodic neither that all modes are visited in each cycle-time (the switching functions do not need to be periodic). Moreover each mode can be visited more than once in each cycle-time from $k T$ and $(k+1) T$ with $k$ any integer. The duty ratio definition (4) is still valid, with $T_{k}=T$ and $\tau_{i, k}$ being the total time duration with the $i$ th mode active within the $k$ th cycle, i.e., between $k T$ and $(k+1) T$.

\section{AVERAGING OVER THE CYCLE-TIME}

Consider a switched system with a constant cycle-time $T$. The duty ratio function of the $i$ th mode can be defined as the moving average over $T$ of the switching function:

$$
d_{i}(t)=\frac{1}{T} \int_{t-T}^{t} q_{i}(s) \mathrm{d} s
$$

with $i=1, \ldots, m$ and $q_{i}(s)=0$ for $s<0$. Clearly, the duty ratio functions $d_{i}(t)$ are bounded (between 0 and 1) piecewise-linear continuous functions. From (6) and since $d_{i}(t)$ is piecewise linear we obtain:

$$
\dot{d}_{i}(t)=\frac{1}{T}\left(q_{i}(t)-q_{i}(t-T)\right),
$$

for almost all $t \in[0,+\infty)$.

If $q_{i}(t)$ for some $i=1, \ldots, m$ are periodic of period $T$, then the corresponding $d_{i}$ will be constant.

Given the switched system (3), by using (6) we can define the following averaged system

$$
\dot{x}_{a v}(t)=\sum_{i=1}^{m} d_{i}(t)\left(A_{i} x_{a v}(t)+B_{i} w(t)\right) .
$$

Since $d_{i}(t)$ are Lipschitz continuous, clearly the averaged system (8) has a unique absolutely continuous solution for $t \in[0,+\infty)[14]$.

So as mentioned above the definition of averaged system is not unique. For instance in [21] the averaged system is defined as the linear time-invariant system

$$
\dot{\tilde{x}}_{a v}(t)=A_{a v} \tilde{x}_{a v}(t)+B_{a v} w(t)
$$

where $A_{a v}$ is a constant matrix satisfying

$$
\left|A_{a v}-\frac{1}{T} \int_{t-T}^{t} \sum_{i=1}^{m} A_{i} q_{i}(s) \mathrm{d} s\right| \leqslant a \beta(T)
$$

and analogously for $B_{a v}$, where $a$ is a positive real number and $\beta:[0,+\infty) \rightarrow[0,+\infty)$ is a class- $\mathcal{L}$ function, i.e. a continuous function converging to zero as its argument grows unbounded. The definition (10) does not require $q_{i}(t)$ to be periodic. On the other hand, it is simple to verify that (8) and (9) coincide if $q_{i}(t)$ for $i=1, \ldots, m$ are periodic of period $T$. Note that by choosing a time-varying averaged matrix defined as in (8), i.e. $A_{a v}(t)=\sum_{i=1}^{m} d_{i}(t) A_{i}$, the argument of the norm in (10) is identically zero. In the case of $T$-periodic switching function in [6] the local stability of the switched system (3) based on that of the time-invariant averaged system (9) is proved, and in [7], [13] the extension to global stability is shown. Moreover in the general case of non-periodic switching functions in [21] it is shown how the stability of the time-invariant averaged system (9) can infer some practical stability results on the switched system (3).

Dealing with the approximation properties between the averaged system (8) and the switched system (3), using similar arguments of [26] it is possible to show that the approximation is of order of the modulation period assuming that the two systems have the same initial condition and that the exogenous input $w(t)$ is bounded, differentiable and with bounded derivative. Some other averaging results have been proved for state and input dependent switching functions, and in next section will be reviewed and recast (particularized) in the switched linear systems framework. Before proceeding with that it is useful to present a generalization of the averaged system definition which exploits a frequency domain interpretation of the moving averaging process.

\section{A. Dynamic phasor model}

The so-called dynamic phasor model can be interpreted as a generalization of the averaged model (8), obtained by including an arbitrary number of harmonics (whereas the averaged model only represents the fundamental). The $\ell$ th phasor (Fourier coefficient) of the state $x$ is defined as:

$$
\langle x\rangle_{\ell}(t)=\frac{1}{T} \int_{t-T}^{t} x(s) e^{-j \ell \omega s} \mathrm{~d} s
$$

with $\omega=\frac{2 \pi}{T}$. It should be noted that the phasors are defined over a moving time window. They are thus time dependent and describe how the harmonics of the solution develop over time. In the sequel for notational simplicity we will omit the indication of the time dependence of the phasors. The time domain signal $x$ is reconstructed on the interval $[t-T, t]$ according to

$$
\mathrm{x}(t, s)=\sum_{\ell=-\infty}^{\infty}\langle x\rangle_{\ell} e^{j \ell \omega(t+s)}, \quad s \in[-T, 0]
$$

note that $x(t) \neq \mathrm{x}(t, s)$ but the equality $x(t+s)=\mathrm{x}(t, s)$ holds for almost all $s \in[-T, 0]$.

The approximation of the variables $x$ with a Fourier series can be seen as a generalized averaged model [17], [18]. The essence in phasor dynamic modeling is to retain only the relatively large Fourier coefficient to capture the interesting behavior of the system. If only the index-zero coefficient is retained for a fast switching system only the low frequency behavior is captured and the result would be the averaged model (8). However when there is ripple in the state variables or when the switching frequency is not high enough we need to add other Fourier coefficients in order to capture, through the dynamic phasor model, the system behavior. 
Consider the equation (11). By using the partial integration we obtain:

$$
\frac{\mathrm{d}}{\mathrm{d} t}\langle x\rangle_{\ell}=\left\langle\frac{\mathrm{d}}{\mathrm{d} t} x\right\rangle_{\ell}-j \ell \omega\langle x\rangle_{\ell} .
$$

Since the state $x$ is bounded continuous and the modeswitching functions $q_{i}$ are bounded piecewise continuous functions over any finite time interval, the phasor coefficients of the product $q_{i} x$ satisfy

$$
\left\langle q_{i} x\right\rangle_{\ell}=\sum_{k=-\infty}^{\infty}\left\langle q_{i}\right\rangle_{\ell-k}\langle x\rangle_{k}
$$

where

$$
\left\langle q_{i}\right\rangle_{\ell}=\frac{1}{T} \int_{t-T}^{t} q_{i}(s) e^{-j \ell \omega s} \mathrm{~d} s .
$$

Note that by comparing (6) and (15) it simply follows that

$$
d_{i}(t)=\left\langle q_{i}\right\rangle_{0}
$$

The switched system dynamic model (13) can then be rewritten in terms of the phasor dynamics:

$$
\begin{aligned}
\frac{\mathrm{d}}{\mathrm{d} t}\langle x\rangle_{\ell}= & -j \ell \omega\langle x\rangle_{\ell}+\sum_{k=-\infty}^{\infty} \sum_{i=1}^{m} A_{i}\left\langle q_{i}\right\rangle_{\ell-k}\langle x\rangle_{k} \\
& +\sum_{k=-\infty}^{\infty} \sum_{i=1}^{m} B_{i}\left\langle q_{i}\right\rangle_{\ell-k}\langle w\rangle_{k}
\end{aligned}
$$

The phasor dynamics (17) can be written in compact form:

$$
\frac{\mathrm{d}}{\mathrm{d} t} \widehat{x}=(-j \omega \mathcal{N}+\widehat{A}) \widehat{x}+\widehat{B} \widehat{w}
$$

where the state and input vectors are the infinite dimensional phasor vectors

$$
\begin{aligned}
\widehat{x} & =\left[\ldots,\langle x\rangle_{1}^{T},\langle x\rangle_{0}^{T},\langle x\rangle_{-1}^{T}, \ldots\right]^{T} \\
\widehat{w} & =\left[\ldots,\langle w\rangle_{1}^{T},\langle w\rangle_{0}^{T},\langle w\rangle_{-1}^{T}, \ldots\right]^{T},
\end{aligned}
$$

$\mathcal{N}=\operatorname{blkdiag}\left(\ldots, 2 I_{n}, I_{n}, 0,-I_{n},-2 I_{n}, \ldots\right)$ is a doubly infinite dimensional block diagonal matrix, and

$$
\begin{aligned}
& \widehat{A}=\sum_{i=1}^{m}\left(I \otimes A_{i}\right) \mathcal{T}\left[\widehat{q}_{i}\right] \\
& \widehat{B}=\sum_{i=1}^{m}\left(I \otimes B_{i}\right) \mathcal{T}\left[\widehat{q}_{i}\right] .
\end{aligned}
$$

where

$$
\widehat{q}_{i}=\left[\ldots\left\langle q_{i}\right\rangle_{1}^{T}\left\langle q_{i}\right\rangle_{0}^{T}\left\langle q_{i}\right\rangle_{-1}^{T} \ldots\right]^{T} .
$$

The transformation $\mathcal{T}$ maps an infinite complex vector $\xi=$ $\left[\ldots, \xi_{1}^{T}, \xi_{0}^{T}, \xi_{-1}^{T}, \ldots\right]$ where $\xi_{k} \in \mathbb{C}$ to a doubly infinite dimensional block Toeplitz matrix according to

$$
\mathcal{T}(\xi)=\left[\begin{array}{ccccc}
\ddots & & \vdots & & \ddots \\
& \xi_{0} I_{n} & \xi_{1} I_{n} & \xi_{2} I_{n} & \\
\cdots & \xi_{-1} I_{n} & \xi_{0} I_{n} & \xi_{1} I_{n} & \cdots \\
& \xi_{-2} I_{n} & \xi_{-1} I_{n} & \xi_{0} I_{n} & \\
\ddots & & \vdots & & \ddots
\end{array}\right]
$$

By comparing (18) with (8) we can say that (8) can be obtained from (18) when the definition (11) is taken by considering only the zero-index component, i.e. only for $\ell=0: x_{a v}(t)=\langle x\rangle_{0}$. In that sense (18) can be interpreted as a generalization of (8). To the best of our knowledge, an analysis of how the dynamic phasor model (17) approximates the behavior of the switched system (3) has not been proposed in the literature yet. Instead, some results on the particular case of state-dependent switching functions have been obtained in [20]. In that paper, by considering a two-modes switched system, the authors assumed statedependent duty ratio functions which are piecewise constant in each modulation period. They analyzed the error between the phasor dynamic model (17) and the truncated system obtained by redefining all (11)-(22) for $\ell \in\{0, \ldots, \Lambda\}$ and $k \in\{-\Lambda, \ldots, 0, \ldots, \Lambda\}$ with a finite $\Lambda$ and by suitably approximating the phasors (15). In particular it is proved that if the origin of the autonomous truncated system is locally exponentially stable, then for each $\epsilon>0$ there exists a modulation period $T_{0}$ such that for any $T \leqslant T_{0}$ the norm of the error between the phasor dynamic model and the truncated system will not exceed $\epsilon$.

So as for the pulse-width modulated systems considered in [20], in many applications the switchings are statedependent. In next section we discuss some averaging results presented in the literature for the case of time-, state-, and input-dependent switching functions.

\section{AVERAGING WITH STATE-DEPENDENT SWITCHINGS}

In order to highlight the possible switching function dependence on the state and on the low-frequency inputs we can specify the switched system (3) as:

$$
\dot{x}(t)=\sum_{i=1}^{m} q_{i}(t, x(t), w(t))\left(A_{i} x(t)+B_{i} w(t)\right)
$$

and the averaged system (8) as

$$
\dot{x}_{a v}(t)=\sum_{i=1}^{m} d_{i}\left(t, x_{a v}(t), w(t)\right)\left(A_{i} x_{a v}(t)+B_{i} w(t)\right),
$$

where

$$
d_{i}(t, x, w)=\frac{1}{T} \int_{t-T}^{t} q_{i}(s, x, w) \mathrm{d} s
$$

for any fixed $x$ and $w$. Assume that the switching function is periodic of period $T$ with respect to the explicit dependence on $t$, i.e.

$$
q_{i}(t, x, w)=q_{i}(t+T, x, w)
$$

for any $x, w, i=1, \ldots, m$ and almost all $t \in[0,+\infty)$. Note that (26) does not imply that the switching function is time-periodic, because in general

$$
q_{i}(t, x(t), w(t)) \neq q_{i}(t+T, x(t+T), w(t+T))
$$

for some $i=1, \ldots, N$. If (26) holds, the duty ratio functions (25) will be time-independent. Then the averaged system will be

$$
\dot{x}_{a v}(t)=\sum_{i=1}^{m} d_{i}\left(x_{a v}(t), w(t)\right)\left(A_{i} x_{a v}(t)+B_{i} w(t)\right),
$$


with the duty ratio functions defined by

$$
d_{i}(x, w)=\frac{1}{T} \int_{0}^{T} q_{i}(s, x, w) \mathrm{d} s
$$

for $i=1, \ldots, m$ and for any $x$ and $w$. In [29] the switching function $q_{i}$ is considered to be dependent on a fast time variable and slow time variable. An averaged time-varying system, called partial, is obtained similarly to (28) by averaging with respect to the fast time variable. By assuming the switching functions to be Lipschitz continuous with respect to the state and to the slow time variable it is shown the closeness of the solution of the switched system with that of the partial averaged system. Additionally though averaging the uniform exponential stability of the actual system is proved.

\section{A. Averaging through dithering}

Continuous pulse-width modulated systems can be interpreted as dithered systems. A dither is a high-frequency signal injected into a system with the objective of modifying its nonlinear behavior. The following example shows how a pulse-width modulated system with two modes can be viewed as a dithered system.

Example 1: Consider a switched system with $\Sigma=\{1,2\}$, and the step function $n: \mathbb{R}-\{0\} \rightarrow\{0,1\}$ defined as

$$
n(z)= \begin{cases}1 & z>0 \\ 0 & z<0\end{cases}
$$

and not defined for $z=0$. In order to control the operating mode of the system a typical choice is

$$
z=k^{T} x(t)+r-\delta(t)
$$

where $k^{T}$ and $r$ are constant and $\delta(t):[0, \infty) \rightarrow \Delta$ (with the bounded set $\Delta \subset \mathbb{R}$ ) is a periodic signal, also called carrier signal and usually chosen as a sawtooth or a triangular. The switching function can be defined as

$$
q_{1}(t, x(t), w(t))=n\left(k^{T} x(t)+r-\delta(t)\right)
$$

almost everywhere, with the nonlinearity $n$ given by (30), $w(t)=r$ and the time dependence of $q_{1}$ representing the high-frequency carrier signal. The switching function of the second mode is defined as

$$
q_{2}(t, x(t), w(t))=1-q_{1}(t, x(t), w(t)),
$$

almost everywhere. The values of $q_{1}$ and $q_{2}$ at the isolated switching instants are defined by the assumption that the switching functions are right-continuous.

More generally, switched dithered systems are characterized by a switching function in the form

$$
q_{i}(t, x(t), w(t)) \triangleq n\left(k_{i}^{T} x(t)+\gamma_{i}^{T} w(t)-\delta_{i}(t)\right)
$$

for $i=1, \ldots, m$, where $k_{i}$ and $\gamma_{i}$ are constant vectors, $\delta_{i}$ are the high-frequency carrier (dither) signals all periodic of period $T$, and $n$ is the step function given by (30). Moreover we assume that (2) holds. Being a $T$-periodic dither signal, the duty ratio functions (25) will not explicitly depend on time and can be written as

$$
d_{i}(x, w)=\frac{1}{T} \int_{0}^{T} n\left(k_{i}^{T} x+\gamma_{i}^{T} w-\delta_{i}(s)\right) \mathrm{d} s
$$

for $i=1, \ldots, m$. The duty ratio functions (35) are also called averaged nonlinearities and their shape will depend on the dither shape [27]. For instance, for sawtooth or triangular signal dithers the averaged nonlinearities will be saturations. In order to have well defined switching functions we need that the switching conditions

$$
k_{i}^{T} x(t)+\gamma_{i}^{T} w(t)=\delta_{i}(t)
$$

for $i=1, \ldots, m$ are verified only at isolated time instants. Note that (25) is defined for constant $x$ and $w$ and then the duty ratio functions will be discontinuous if the dither signal has zero-slope time intervals. Moreover there are dither signals for which the corresponding duty ratio functions will be continuous but not Lipschitz continuous [27].

By assuming that the averaged nonlinearity and the exogenous input are Lipschitz continuous, the averaging theorem in [26] allows to conclude that the averaged system (28) with duty ratio functions given by (35) will have a unique absolutely continuous solution. Moreover, if the dither frequency is sufficiently high, the behavior of the switched system (23) with the switching functions (34) will be qualitatively the same as the averaged system over any finite time horizon, provided that the two systems have the same initial condition. Note that the theorem in [26] can be also applied to the more general class of nonsmooth systems in the form (23) and (34) with $n$ being nonsmooth nonlinearities of bounded variation, its argument being a Lipschitz continuous function, and with the terms $A_{i} x+B_{i} w$ in (23) being generalized as Lipschitz continuous, eventually time-dependent, functions.

Dealing with stability issues, in [25] by neglecting the exogenous input $w$ and by generalizing the terms $A_{i} x$ in (23) with continuous functions of the state, it is proved that if the averaged system is asymptotically stable then the switched system is practically stable for any dither period lower than a suitable value. In [27] a detailed discussion on the implications of the dither shape on a similar type of stability inference is presented.

\section{B. Generalized averaging for hybrid systems}

In the recent literature the averaging results for switched systems have been extended to a more general class of hybrid systems [28]. Those results are here recast in the simpler case of switched linear systems. In [28] the homogeneous case is considered, then the switched system of interest is given by (23) without $w$ and the corresponding averaged system is (28)-(29) without $w$. Let define the function

$$
\eta(\vartheta, x)=\int_{0}^{\vartheta} \sum_{i=1}^{m}\left[q_{i}(\xi, x)-d_{i}(x)\right] A_{i} x \mathrm{~d} \xi
$$

Note that the function $\eta(\vartheta, x)$ is bounded and Lipschitz continuous. By particularizing the averaging theorem in [28] to the case of switched linear systems, one can conclude 
the closeness of solutions between the switched and the averaged system if the initial conditions of the two systems are sufficiently close to each other. So as in [26] and in [29] the closeness of the solutions over a finite time horizon does not require any stability assumption. Moreover, by introducing some stability conditions on the averaged system it is possible to conclude the practical stability of the switched one.

\section{CONCLUSions}

Averaging for nonsmooth systems is a research field which still needs many definitive answers. By recasting several approaches presented in the literature, this paper have shown that also by considering the simple case of switched linear systems there are many interesting open issues to be addressed. For instance it could be of interest to prove the closeness of solutions between the switched system and the averaged system for the phasor dynamic model. One should expect that the approximation between the switched and the averaged system reduces when a larger number of harmonics is considered. That opens another interesting line of research. Indeed many practical examples show that the bound on closeness of switched and averaged solutions is much smaller than its theoretical prediction: to look for techniques able to find more realistic bounds is an interesting challenge. Also, the possible usefulness of a time-varying (but smooth) averaged system for the stability analysis of the (nonsmooth) switched system needs more justifications. Other directions of future research could be to investigate averaging for switched models with multiple modulation periods or with non-periodic state-dependent switching functions.

\section{REFERENCES}

[1] D. Liberzon, Switching in System and Control. Boston, Massachussetts, USA: Birkhäuser, 2003.

[2] J. G. Kassakian, M. F. Schlecht, and G. C. Verghese, Principles of power electronics. Reading, MA: Prentice-All, 1991.

[3] R. D. Middlebrook and S. Cuk, "A general unified approach to modelling switching converter power stages," in Proc. of the IEEE Power Electronics Specialists Conference, Cleveland, Ohio, USA, 1976, pp. 73-86.

[4] J. Sun and H. Grotstollen, "Averaged modelling of switching power converters: Reformulation and theoretical basis," in Proc. of the IEEE Power Electronics Specialists Conference, Toledo, Spain, 1992, pp. $1165-1172$.

[5] M. Porfiri, D. G. Roberson, and D. J. Stilwell, "Tracking and formation control of multiple autonomous agents: a two level consensus approach," Automatica, vol. 43, no. 8, pp. 1318-1328, 2007.

[6] D. J. Stilwell, E. M. Bollt, and D. G. Roberson, "Sufficient conditions for fast switching synchronization in time-varying network topologies," SIAM Journal on Applied Dynamical Systems, vol. 5, no. 1, pp. 140-156, 2006.

[7] M. Porfiri and F. Fiorilli, "Global pulse synchronization of chaotic oscillators through fast-switching: theory and experiments," Chaos, Solitons and Fractals, vol. 41, no. 1, pp. 245-262, 2009.

[8] X. Shen, J. Zhang, E. J. Barth, and M. Goldfarb, "Nonlinear averaging applied to the control of pulse-width modulated (PWM) pneumatic systems," in Proc. of the American Control Conference, Boston, Massachussetts, USA, 2004, pp. 4444-4448.

[9] A. K. Gelig and A. N. Churilov, Stability and Oscillations of Nonlinear Pulse-modulated Systems. Boston, Massachussetts, USA: Birkhäuser, 1998.

[10] B. Sedghi, B. Srinivasav, and R. Longchamp, "Control of hybrid systems via dehybridization," in Proc. of the American Control Conference, Anchorage, Alaska, USA, 2002, pp. 692-697.
[11] R. Marquez, E. Altman, and S. Solé-Álvarez, "Time-averaging of high-speed data transfer protocol," IEEE Transactions on Automatic Control, vol. 50, no. 12, pp. 2065-2069, 2005.

[12] H. Sira-Ramírez, M. Zribi, and S. Ahmad, "Pulse width modulated control of robotic manipulators," in Proc. of the American Control Conference, Honolulu, Hawaii, 1990, pp. 336-367.

[13] M. Porfiri and F. Fiorilli, "Experiments on node-to-node pinning control of Chua's circuits," Physica D, vol. 239, no. 8, pp. 454-464, 2010.

[14] H. Khalil, Nonlinear Systems, 3th ed. Upper Saddle River, New Jersey: Prentice Hall, 2002.

[15] M. Khapaev, "On the method of averaging and on certain problems connected with averaging," Differential Equations, vol. 2, no. 5, pp. 310-314, 1966

[16] D. D. Bainov and S. D. Milusheva, "Justification of the averaging method for a system of functional-differential equation with variable structure and impulses," Applied Mathematics and Optimization, vol. 16, pp. 19-36, 1987.

[17] S. R. Sanders, J. M. Noworolski, X. Z. Liu, and G. C. Verghese, "Generalized averaging method for power conversion circuits," IEEE Transactions on Power Electornics, vol. 6, no. 2, pp. 251-259, 1991.

[18] V. Caliskan, G. Verghese, and A. Stanković, "Multifrequency averaging of dc/dc converters," IEEE Transactions on Power Electronics, vol. 14 , no. 1 , pp. 124-132, 1999.

[19] M. Porfiri, D. G. Roberson, and D. J. Stilwell, "Fast switching analysis of linear switched systems using exponential splitting," SIAM Journal of Control Optimization, vol. 47, no. 5, pp. 2582-2597, 2008.

[20] S. Almér and U. Jönsson, "Harmonic analysis of pulse-width modluated systems," Automatica, vol. 45, no. 4, pp. 851-862, 2009.

[21] W. Wang and D. Nešić, "Input-to-state set stability and averaging of linear fast switching systems," IEEE Transactions on Automatic Control, vol. 55, no. 5, pp. 1274-1279, 2010.

[22] J. Ezzine and A. H. Haddad, "Error bounds in the averaging of hybrid systems," IEEE Transactions on Automatic Control, vol. 34, no. 11, pp. 1188-1192, 1989.

[23] G. Zames and N. A. Shneydor, "Dither in non-linear systems," IEEE Transactions on Automatic Control, vol. 21, no. 5, pp. 660-667, 1976.

[24] B. Lehman and R. Bass, "Extensions of averaging theory for power electronics systems," IEEE Transactions on Power Electronics, vol. 11, no. 4 , pp. 542-553, 1996.

[25] A. Teel, L. Moreau, and D. Nešić, "Input-to-state set stability of pulse width modulated systems with disturbances," Systems and Control Letters, vol. 51, no. 1, pp. 23-32, 2004.

[26] L. Iannelli, K. Johansson, U. Jönsson, and F. Vasca, "Averaging of nonsmooth systems using dither," Automatica, vol. 42, no. 4, pp. 669676, 2006.

[27] - "Subtleties in the averaging of a class of hybrid systems with applications to power converters," Control Engineering Practice, vol. 18 , no. 8, pp. 961-975, 2008.

[28] A. Teel and D. Nešić, "Averaging theory for a class of hybrid systems," Dynamics of Continuous, Discrete and Impulsive Systems, vol. 17, no. 6, pp. 829-851, 2010.

[29] M. Grammel and I. Maizurna, "Exponential stability and partial averaging," Journal of Mathematical Analisys and Applications, vol. 283, no. 1, pp. 276-286, 2003.

[30] S. J. Liu and M. Krstic, "Continuous-time stochastic averaging on the infinite interval for locally lipschitz systems," SIAM Journal of Control and Optimization, vol. 48, no. 5, pp. 3589-3622, 2010.

[31] M. Porfiri, D. Stilwell, and E. Bollt, "Synchronization in random weighted directed networks," IEEE Transactions on Circuits and Systems, vol. 55, no. 10, pp. 3170-3177, 2008.

[32] M. Porfiri, D. Stilwell, E. Bollt, and J. Skufca, "Random talk: random walk and synchronizability in a moving neighborhood network," Physica D, vol. 224, no. 1-2, pp. 102-113, 2006.

[33] M. Porfiri and D. Stilwell, "Consensus seeking over weighted directed graphs," IEEE Transactions on Automatic Control, vol. 52, no. 9, pp. 1767-1773, 2007.

[34] I. Belykh, V. Belykh, and M. Hasler, "Blinking model and synchronization in small-world networks with a time-varying coupling," Physica D, vol. 195, no. 1-2, pp. 188-206, 2004.

[35] T. Donchev and I. Slalov, "On the limit of the solution set of singularly perturbed control differential inclusions," in Proc. of the IEEE Conference on Decisions and Control, Phoenix, Arizona, USA, 1999, pp. 3340-3345. 